\title{
Topic-the effect of three cavity disinfectants (chlorhexidine gluconate-based. Consepsis; benzalkonium chlorite-based, Tubulicid red; sodium hypochlorite based-Chlorcid V on the self - etch dentine bonding agent ( Adeper Easy One,3M ESPE ) under SEM.
}

\author{
${ }^{1}$ Dr. Navdheeraj Pattanaik, ${ }^{2}$ Dr. Manoj Chandak, \\ ${ }^{I}$ M.D.S, Lecturer Department of Conservative and Endodontics, Sharad Pawar Dental College, Wardha, \\ Maharastra,India, \\ ${ }_{2}$ M.D.S, Professor and H.O.D, Department of Conservative and Endodontics, Sharad Pawar Dental \\ College, Wardha, Maharastra,India
}

\begin{abstract}
The purpose of this study was to determine the effect of three cavity disinfectants (chlorhexidine gluconate-based. Consepsis; benzalkonium chlorite-based, Tubulicid red; sodium hypochlorite based-Chlorcid $V$ on the self-etch dentine bonding agent (Adeper Easy One,3M ESPE) under SEM. Method and materials: 30 Class $V$ cavity preparations were prepared on the buccal surfaces of extracted mandibular molars with occlusal margins at the enamel and gingival margins in cementum then they were divided into 3 experimental groups-A,B and $C$. In the experimental groups, cavities were treated with combinations of one of the three cavity disinfectants with Adeper Easy One, self -etch dentine bonding agent. After the cavity preparations were restored with resin composite Filtek Z $35 O$ (3M ESPE), specimens were thermocycled, , and sectioned the toothresin composite interface of the sectioned specimens was examined under scanning electron microscopy. Results: Consepsis and Tubilicid red specimens shows the resin-tag formation whereas Chlorcid V exhibited marginal gap formations at the tooth-resin composite interface. Conclusion: $2 \%$ chlorhexidine gluconate and benzalkonium chlorite can be used as cavity disinfectants with Adeper Easy One SE bonding agent. However sodium hypochlorite is not an appropriate disinfectant to use with this bonding system as it showed marginal gap formation.
\end{abstract}

Keywords: cavity disinfectants, self etch dentine bonding agent, chlorhexidine gluconate, benzalkonium chlorite and sodium hypochlorite.

\section{Introduction}

Achievement of a consistently reliable, gap-free, and complete intra- oral attachment of resin composite to dentine would profoundly change the clinical practice of operative dentistry ${ }^{1}$. The efficacy of dentine bonding systems have been remarkably improved by introduction of dentine primer by Munksgaard and Asmussen in 1984.They stated that pre-treatment of dentine with an aqueous mixture of glutaraldehyde and hydroxyethyl methacrylate ( HEMA) promote the bonding efficacy of resin materials ${ }^{2}$. According to Nakabayshi formation of hybrid layer is essential for high bond strength and resin tags formation ${ }^{3}$.

But it is well-documented that the setting reaction of resin composites may lead to the formation of contraction gap at the tooth-restoration interface. This gap can result in the passage of bacteria, fluids, or ions between the cavity wall and the resin composite, a process defined as microleakage ${ }^{4}$.

The problems associated with microleakage can be magnified by incomplete sterilization of the preparation as a consequence of failure to mechanically remove infected tooth structure ${ }^{5}$. Histological and bacteriologic studies have shown that only a small proportion of the teeth are sterile after cavity preparation. A number of studies have demonstrated that the bacteria left in the dentin of a cavity preparation could maintain their activity for a long time ${ }^{6}$.

\section{Brannstrom}

Indicated that residual bacteria in a cavity preparation can multiply from within the smear layer, even in the presence of a good seal from the oral cavity. This can be a source of bacterial toxins, which can diffuse or even cause recurrence of the caries process ${ }^{7}$. The application of disinfectants after cavity preparation and before tooth restoration is gaining wider acceptance in order to eliminate the potential risks due to bacterial activity ${ }^{8}$.

Studies have depicted that a number of antibacterial solutions such as Chlorhexidine, Sodium hypochlorite, fluoride based solutions Benzalkonium chloride can be used as cavity disinfectants to eliminate the residual bacteria from the prepared cavity. 
However, there is concern about the use of cavity disinfectants with dentin-bonding agents, since they may alter the ability of hydrophilic resin to seal the dentin ${ }^{9}$. C Contrary to this concern, it has been suggested that cavity disinfectants can improve the sealing ability' of dentin-bonding agents by remoistening the cavity prior to placing a dentin bonding agent that bonds to damp tooth structure ${ }^{10}$.

The purpose of this study was to evaluate and compare the the effect of three cavity disinfectants (chlorhexidine gluconate-based ( Consepsis); benzalkonium chlorite-based( Tubulicid red)

Sodium hypochlorite based(Chlorcid V) on the self -etch dentine bonding agent ( Adeper Easy One,3M ESPE ) under SEM.

\section{Methodology}

A total 30 freshly extracted human mandibular permanent molars were selected for the study. Immediately after extraction, the teeth were kept in hydrogen peroxide solution washed under running tap water for 15 minutes each. Later they were cleaned in pumice and stored in normal saline at $40^{\circ} \mathrm{C}$ until use.

Class V cavity were prepared on the buccal surface of each tooth with a cylindrical diamond bur -Dianfong:SR12, Standardized class V cavity preparation were done by preparing the cavity that were 2 mm wide (occlusogingivally) ,1.5mm deep, $3 \mathrm{~mm}$ long (mesio-distally ) paralleling the cement enamel junction. The gingival half of the preparation was extended $0.5 \mathrm{~mm}$ below the CEJ.

Each preparation was rinsed with distilled water for 20 seconds and dried for 20 seconds. Then the teeth were randomly divided into 3 Groups-A, B and C.

Group A-consisted of 10 class V cavities, the chlorohexidine based cavity disinfectant CONSPESIS, Ultradent, USA was applied on the cavity walls and on the floor using disposable micro applicators for 20 seconds and excess disinfectant was removed by 5 seconds of light air. Then Self -Etch adhesive Adeper Easy One ( $3 \mathrm{M}$ ESPE, Germany) was applied to the preparation with a brush, spread gently with air and cured for 20 seconds. The cavity preparations were restored with resin composite- FILTEK Z 350 XT (3M ESPE,USA ) by light curing for 60 seconds.

Group B-the benzalkonium chloride cavity disinfectant- Tubulicid red, Dental therapeutics AB, Sweden was applied on the cavity walls and on the floor. Then Self -Etch adhesive Adeper Easy One ( 3M ESPE, Germany) was applied and cured .The cavity preparations were restored with resin composite- FILTEK Z 350 . Group A and B.

Group C- Chlor- Cid V, Ultradent, USA was applied and the same procedure was repeated as if in

All the teeth were stored in distilled water for 24 hours, at $37^{\circ} \mathrm{C}$ and then subjected for thermal cycling with 1000 cycles between water baths of $50^{\circ} \mathrm{C}-550^{\circ} \mathrm{C}$ with a dwell time of 30 seconds.

The samples were then subjected for SEM evaluation-. The teeth were sectioned mesiodistally and buccolingually in approximate center of restorations with a low-speed diamond saw (Isomet, Buehler). Sectioned surfaces were treated with $35 \%$ phosphoric acid (Ultradent) for 10 seconds and rinsed with water stream for 10 seconds.

The specimens were then dehydrated in ascending grades of ethyl alcohol (70\%, 80\%, 90\% and $100 \%)$ and vaccum dried. Later on the samples were coated with $20 \mathrm{~nm}$ of gold and viewed under SEM (JEOL/ JSM, Model no.5400, Japan).

Group A-SEM evaluation of the speciemens at magnification X 500, X1000 and X 2000-shows the appearance of resin tags.

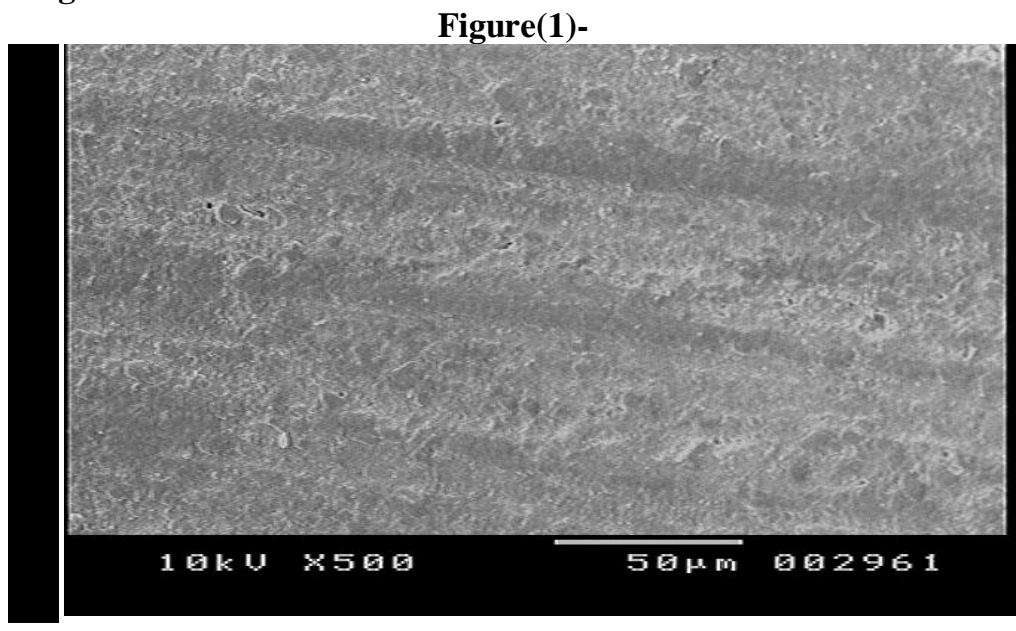


Figure(2)-

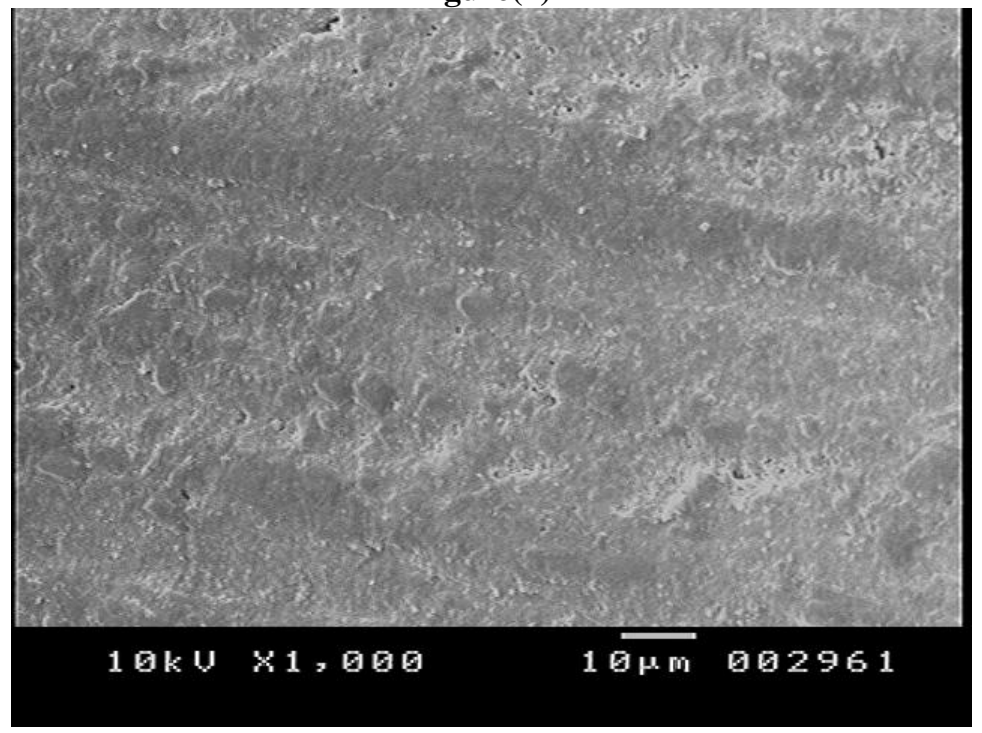

Figure(3)-

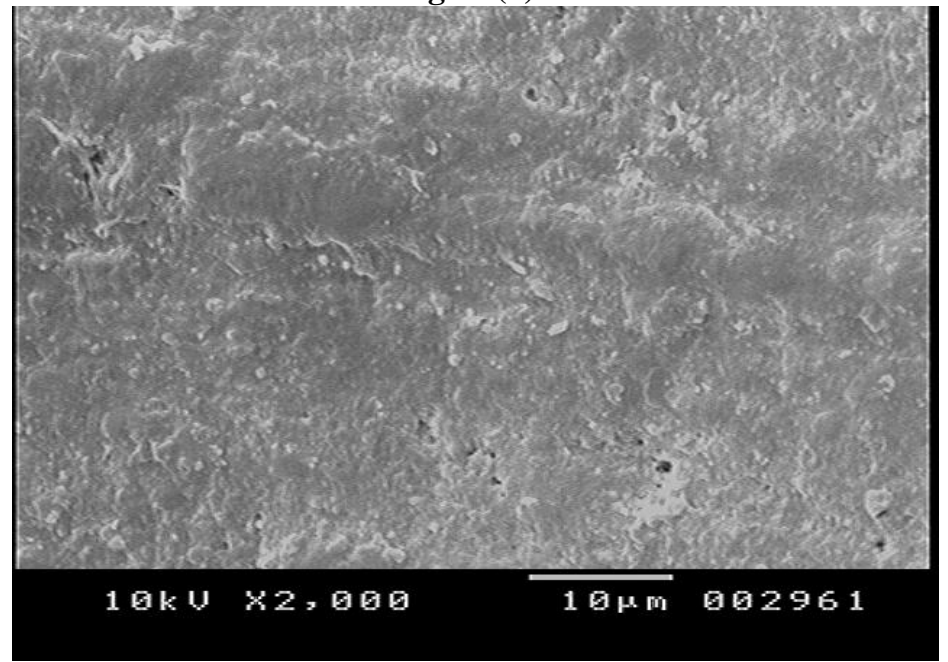

Group B-SEM evaluation of the speciemens at magnification X 500, X1000 and X 2000-also shows the appearance of resin tags.

Figure (1)-

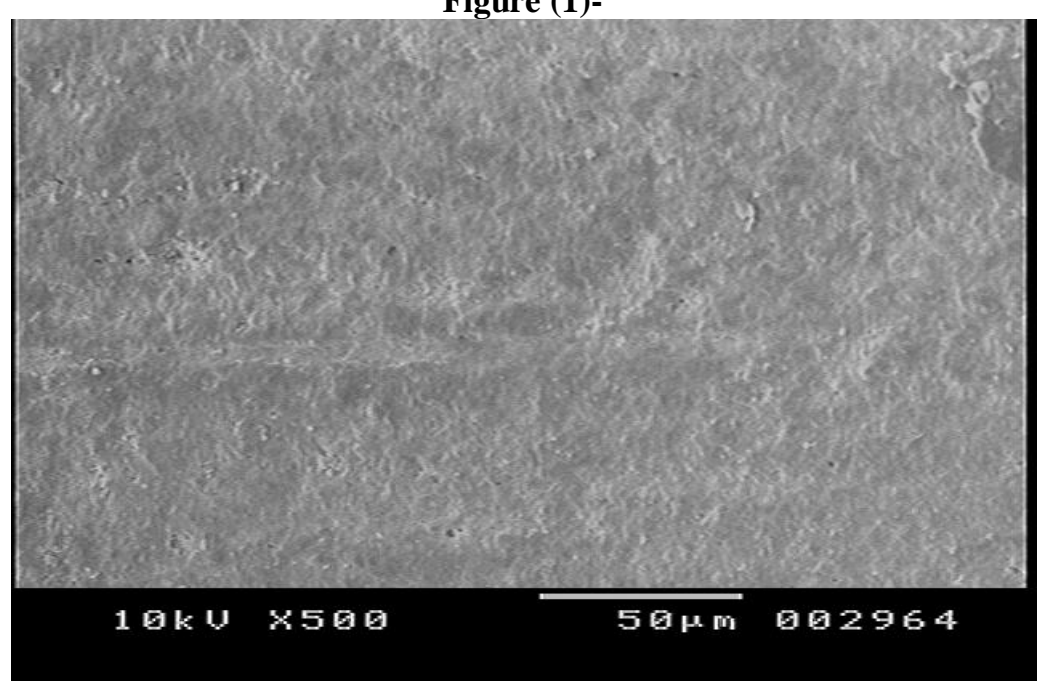


Figure (2)-

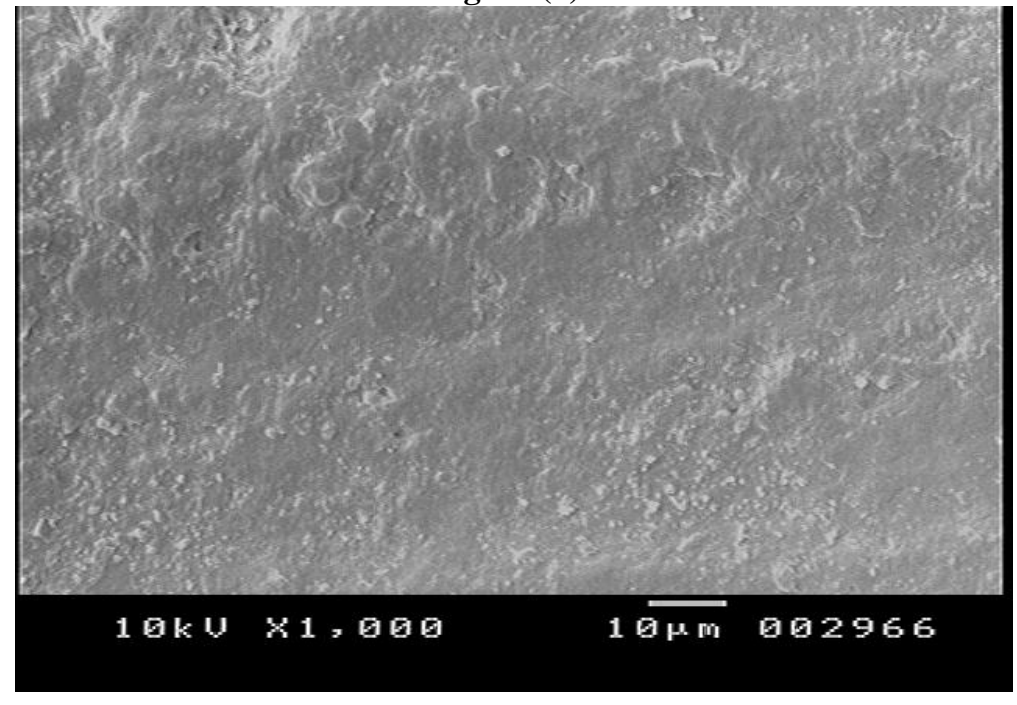

Figure (3)-

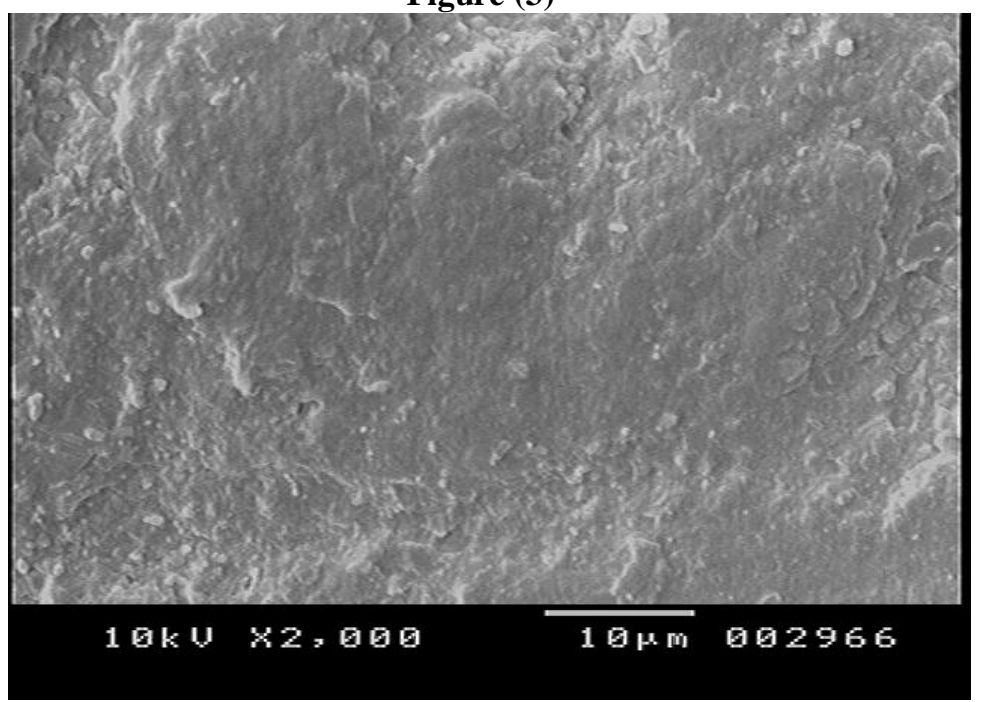

Group C-SEM evaluation of the speciemens at magnification X 500, X1000 and X 2000-also shows the appearance marginal gap at tooth composite interferace. Figure (1)-

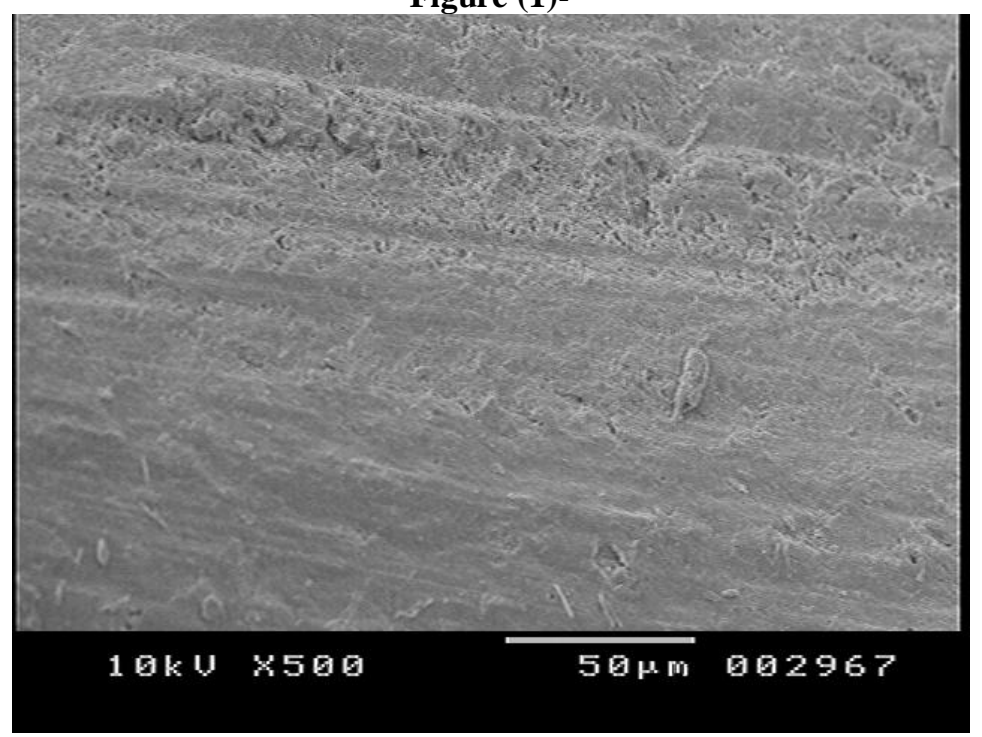



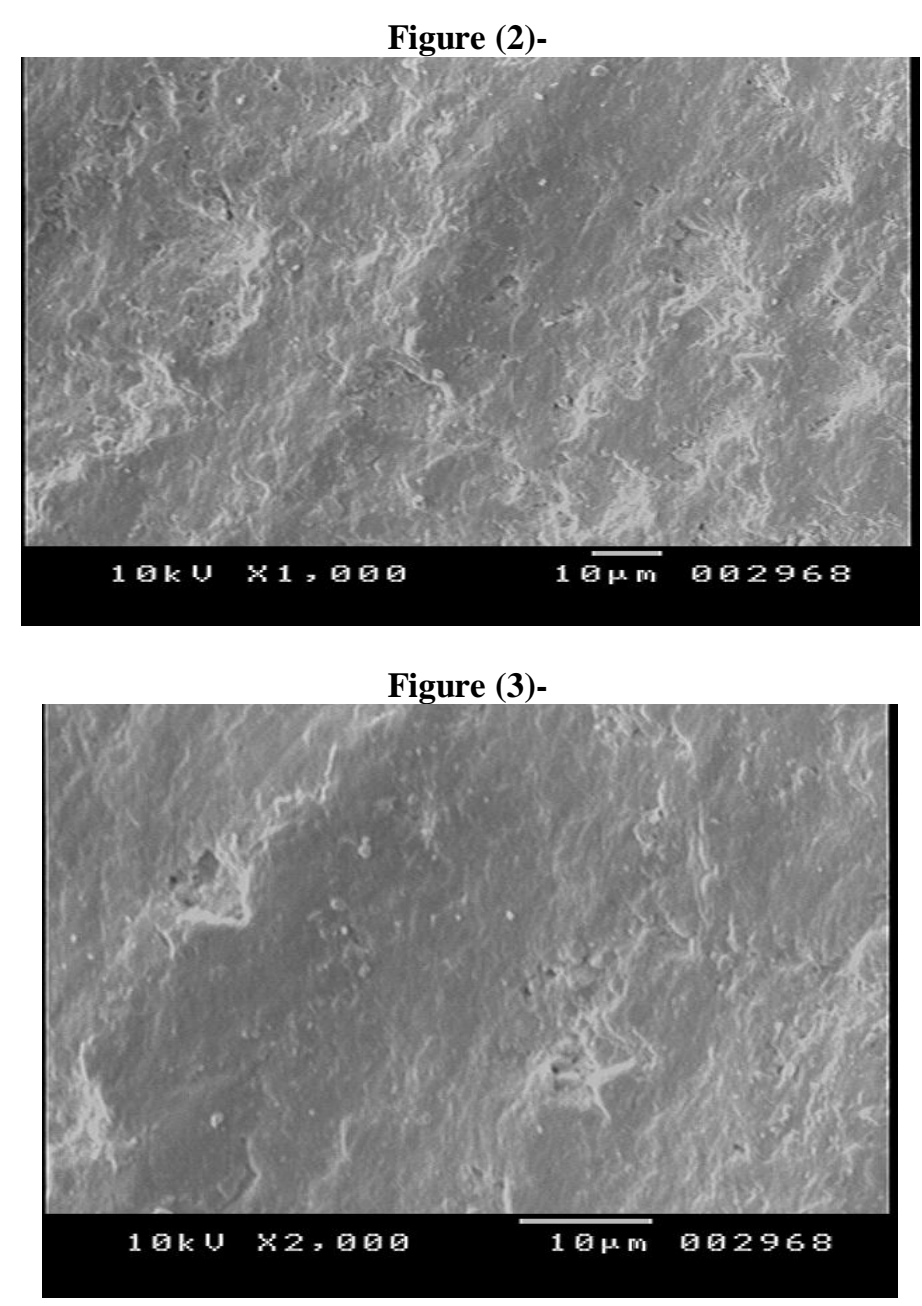

\section{Discussion}

It is clinically very important to enhance the adhesion between the dentine and the adhesive resin, because such improved adhesive strength not only leads to better retention of restorations but prevents marginal leakage thus reducing the chances of developing secondary caries ${ }^{11}$.

In 1972 Brannstorm et al in his study reported the prevalence of high frequency of bacteria beneath the composite restorations the two main factors considered are chemical irritation by the material and poor adaptation ${ }^{12}$. Historically, it was suggested that dentin should be sterilized before the placement of any restorative material. Many chemicals, such as silver nitrate precipitated with eugenol, thymol, and potassium ferrocyanide, had been proposed for this purpose ${ }^{12}$.Today, it is known that these chemicals are irritating to the pulp when applied to the dentin surface.

Consepsis used in our study contains $2 \%$ chlorhexidine gluconate and has been reported to have better antimicrobial activity. According to J C Meirs and JC Kresin chlorhexidine washes did not remove the smear layer but did modify its appearance by removing loose smear debris ${ }^{8}$.

Gultz et al (1995) who had compared the antimicrobial activities of different cavity disinfectants found that Consepsis solution was superior as compared with other cavity disinfectants as. Consepsis solution when used with Clearfii SE Bond and Prompt L-Pop demineralize the dentin and envelop the collagen fibers and hydroxylapatite crystals ${ }^{13}$. The scanning electron microscopic observations of their study revealed the presence of resin -tags in the Consepsis treated group.However, the results of our study are conflicting with other studies.

Chlorhexidine solution had an adverse effect on Syntac and Prime \& Bond and produced significantly higher microleakage when used with these bonding systems ${ }^{14}$.

The Tubulicid Red used in our study contains ethylene diamine tetracitic acid (EDTA) for the removal of smear layer and benzalkonium chloride, which has an antibacterial effect. It also contains a high concentration of sodium fluoride and has been claimed to accomplish three goals in one treatment -cleaning, disinfection, and impregnation with $20 \%$ reduction in dentinal permeability ${ }^{15}$. When this solution used in combination with dentine bonding systems it bond micromechanically to intertubular dentine. The bonding agents adhere to the 
intertubular dentine while the dentinal orifices remain closed by fluoridated smear plugs and thus prevents the influx of irritants to the pulp.

Chlorcid V when used as cavity disinfectants the SEM evaluation shows the presence of marginal gap at the tooth composite interferace. According to R.Frankerberger et al $\mathrm{NaOCl}$ pre treatment has detrimental effects on the dentine bonding performance of the dentine adhesive system and thus resulted in lower bond strength and worse marginal adaptation due to the unhindered free shrinkage towards the bonded area ${ }^{16}$.

Mirela Sanae Shinohara et al stated that dentin collagen does not contribute to dentin adhesion and may even interfere with the bonding mechanisms because of the fragile structure of collagen fibers after etching 17. Haller et al (2002) stated that the application of $10 \% \mathrm{NaOCl}$ considerably influences adhesive systems containing ethanol and acetone by interfering with the wettability of dentin surfaces ${ }^{18}$.

Study by Lai et al (2001) suggested the presence of the reactive residual free-radicals in dentin treated with sodium hypochlorite may compete with the propagating vinyl free-radicals generated during light activation of the adhesive leads to increase microleakage ${ }^{19}$.

Therefore, it can be concluded from our study that the use of cavity disinfectants with resin composite restorations appears to be material specific, with regard to interaction with the ability of various dentin bonding systems to seal dentin.

Clinical significance-The application of disinfectants after cavity preparation and before the restoration is gaining acceptance. This study opens the perspective further research of the use of cavity disinfectants in dentistry as it behaves in the oral environment after cavity preparation. So it would prudent to research the performance of the cavity disinfectant in long term clinical study

\section{Conclusion}

The following conclusions can be drawn from the results of this study:

- Conspesis and Tubulicid Red can be used as cavity disinfectants with Adeper Easy One ( Self Etch Adhesive) as it results in the formation of resin tags at the tooth composite interference.

- Whereas Chlorcid V cannot be used as cavity disinfectants with Adeper Easy One ( Self Etch Adhesive) as it leads to the formation of marginal gap with no resin tags formation at the tooth composite interference.

\section{Acknowledgment}

The authors are thankful to Mr. Kenneth Larsson of Dental Therapeutics AB, Sweden, Mr. Nathan D Johnson of Ultradent USA, , for providing cavity disinfectants related to the study. For SEM study us whole heartedly thanks to Department Of Metallurgy, VNIT Nagpur, India

\section{References}

[1]. P.R.Walshaw,D.McComb .SEM Evaluation of resin-dentin interference with proprietary bonding agent in human subjects. J Dent Res. 1994 May; 73(5):1079-87.

[2]. H.Chigara, W.Yutikani ,T.Hasegawa ,K.Itoh.Self-etching dentine primers containing Phenyl-P. J Dent Res May 1994; 73(5): 10881095 .

[3]. N.Nakabayshi. Bonding of restorative materials to dentine: the present status in Japan. Int Dent J. 1985 Jun; 35(2):145-54.

[4]. Kidd EA.Microleakage: a review. J Dent. 1976 Sep; 4(5):199-206.

[5]. Gultz.J, Boylan R, Schers W. Antibacterial activity of cavity disinfectants. Gen Dent. 1999 Mar-Apr; 47(2):187-90.

[6]. Besic C. F. The fate of bacteria sealed in dental cavities. J Dent Res October 1943; 22(5): 349-354

[7]. Brannstorm M, Nyborg Hilding. Cavity treatment with a microbial fluoride solution: Growth of the bacteria and the effect on the pulp J Prosthet Dent. 1973 Sep; 30(3):303-10.

[8]. Meirs JC, Kresin JC. Cavity disinfectants and dentine bonding. Oper Dent. 1996 Jul-Aug; 21(4):153-9.

[9]. M Turkun, L S Turkun, Z Equire. Is an antibacterial adhesive systems more effective than cavity disinfectants? Am J Dent. 2006 Jun;19(3):166-70.

[10]. Murat Turkun, Shebnem Turkum ,Kalender Atkan . Effect of cavity disinfectants on the sealing ability of the non-rinsing dentine bonding resins. Quintessence Int. 2004 Jun;35(6):469-76.

[11]. Yuki Wakabayshi, Yasuhiro Kondou, Kazoumi Suzuki. Effect of dissolution of collagen on adhesion to dentine. Int J Prosthodont. 1994 Jul-Aug; 7(4):302-6.

[12]. Brannstorm M, Nyborg H,. Pulpal reaction to composite resin restoration.J Prosthet Dent. 1972 Feb; 27(2):181-9.

[13]. Gultz.J, Boylan R, Schers W. Antibacterial activity of cavity disinfectants. Gen Dent. 1999 Mar-Apr; 47(2):187-90.

[14]. O Tulunoglu, H.Ayhan, Olmez A, H.Bodur. The effect of cavity disinfectants on the microleakage in dentine bonding system. J Clin Pediatr Dent. 1998 Summer; 22(4):299-305.

[15]. Brannstorm M .Reducing the risk of sensitivity and pulpal complication after the placement of crowns and fixed partial dentures. Quintessence Int. 1996 Oct; 27(10):673-8.

[16]. Frankerberger R, Kramer N, H Oberschachtsiek, Petschelt A. Dentine bond strength and marginal adaptation after NaOcl pre tereatment. Oper Dent. 2000 Jan-Feb; 25(1):40-5.

[17]. Sanae Mirela, Shinorhara Mirela, Barbieri Ana Karina .The effect of sodium hypochlorite on the microleakage of composite resin restoration using three adhesive systems. J Adhes Dent. 2004 Summer; 6(2):123-7.

[18]. B Haller. Recent developments in dentine bonding. Am J Dent. 2000 Feb;13(1):44-50

[19]. Lai SCN, Mak YF, Toledano M. Reversal of compromised bonding to oxidized etched dentine. J Dent Res 2001, 80(10):1919-1924. 